\title{
Power Allocation Optimization: Linear Precoding Adapted to NB-LDPC Coded MIMO Transmission
}

\author{
Tarek Chehade, ${ }^{1,2}$ Ludovic Collin, ${ }^{1,2}$ Philippe Rostaing, ${ }^{1,2}$ \\ Emanuel Radoi, ${ }^{1,2}$ and Oussama Bazzi ${ }^{3}$ \\ ${ }^{1}$ Université Européenne de Bretagne, 5 Boulevard Laënnec, 35000 Rennes, France \\ ${ }^{2}$ Université de Brest, CNRS, UMR 6285 Lab-STICC, 6 avenue Victor Le Gorgeu, CS 93837, 29238 Brest Cedex 3, France \\ ${ }^{3}$ Department of Physics and Electronics, Faculty of Sciences I, Lebanese University, Hadath, Beirut, Lebanon
}

Correspondence should be addressed to Tarek Chehade; tarek.chehade@univ-brest.fr

Received 19 September 2014; Revised 31 December 2014; Accepted 14 January 2015

Academic Editor: Yan Zhang

Copyright (c) 2015 Tarek Chehade et al. This is an open access article distributed under the Creative Commons Attribution License, which permits unrestricted use, distribution, and reproduction in any medium, provided the original work is properly cited.

In multiple-input multiple-output (MIMO) transmission systems, the channel state information (CSI) at the transmitter can be used to add linear precoding to the transmitted signals in order to improve the performance and the reliability of the transmission system. This paper investigates how to properly join precoded closed-loop MIMO systems and nonbinary low density parity check (NBLDPC). The $q$ elements in the Galois field, GF( $q$ ), are directly mapped to $q$ transmit symbol vectors. This allows NB-LDPC codes to perfectly fit with a MIMO precoding scheme, unlike binary LDPC codes. The new transmission model is detailed and studied for several linear precoders and various designed LDPC codes. We show that NB-LDPC codes are particularly well suited to be jointly used with precoding schemes based on the maximization of the minimum Euclidean distance (max- $\left.d_{\min }\right)$ criterion. These results are theoretically supported by extrinsic information transfer (EXIT) analysis and are confirmed by numerical simulations.

\section{Introduction}

Multiple-input multiple-output (MIMO) schemes have become unavoidable for transmission systems looking for increased throughput and improved reliability. Many methods have lately been developed to exploit the diversity offered by multiantenna systems like antenna distribution or space/polarization isolation [1], space-time block codes (e.g., Alamouti scheme) that do not require any channel state information at the transmitter side (Tx-CSI), or power allocation optimization techniques, also called precoding, that require a full or partial Tx-CSI. In this paper, we focus on NB-LDPC codes that are widely used because of their effectiveness. One advantage of NB-LDPC codes over binary LDPC codes is that nonbinary codes can match very well the underlying modulation, so symbol-to-bit conversion at the receiver can be avoided. The results in [2] confirm the advantages of using NB-LDPC codes to match the underlying high order modulations.

To the best of our knowledge, the MIMO linear precoding performances are typically evaluated for uncoded MIMO systems. In this paper, we examine the association of the precoding scheme with channel coding in order to finally determine a power allocation optimization solution that adapts a block of linear precoding to a NB-LDPC coded MIMO transmission, where the Belief Propagation algorithm is used for decoding.

We implement this new scheme using linear precoders, having diagonal or nondiagonal structure, and we study the impact of the application of different known precoders on two system configurations $((2,2)$ and $(2,4) \mathrm{MIMO})$. We investigate, by means of an EXIT analysis, how to take advantage of the precoding techniques in a MIMO system that uses NBLDPC codes and we show why some associations could be harmful and others beneficial.

The main contributions of this paper are listed as follows:

(i) a proposed scheme associating NB-LDPC codes to MIMO precoding systems with adequate correspondence between the $\mathrm{GF}(q)$ elements and the $q$ received constellation points;

(ii) a comparison between different precoder types and a study of the gain/loss in the different cases; 
(iii) a theoretical EXIT analysis in order to predict the performance of different precoders with the NBLDPC codes;

(iv) a confirmation by numerical simulations, in terms of bit error rate (BER), of the performance of the proposed power allocation optimization scheme.

This paper is organized as follows. In Section 2 the system model is described with the eigenmode representation. LDPC codes are introduced in Section 3. Linear precoding techniques are presented in Section 4. Section 5 provides a detailed description of the new proposed scheme; Section 6 presents the EXIT analysis, while the simulation results are given in Section 7. Finally, some conclusions are drawn in Section 8 .

The notations used in this paper are as follows. Matrices and vectors are denoted by symbols in boldface, and $(\cdot)^{T}$ and $(\cdot)^{H}$ represent complex transpose and Hermitian, respectively. $\mathbf{I}_{m}$ denotes the $m \times m$ identity matrix and $\operatorname{diag}(\mathbf{x})$ denotes a diagonal matrix with $\mathbf{x}$ on its main diagonal. $E[\cdot]$ denotes statistical expectation. $\mathbf{X}\left[i_{1}: i_{2}, j_{1}: j_{2}\right]$ denotes a submatrix obtained by extracting rows $i_{1}$ through $i_{2}$ and columns $j_{1}$ through $j_{2}$ from a matrix $\mathbf{X}$. If no specific range appears at the row or column position in notation, then all rows or columns are considered to constitute the submatrix. $[\mathbf{X}]^{i j}$ denotes the $(i, j)$ th element of a matrix $\mathbf{X}$. $\|\mathbf{x}\|$ denotes the 2-norm of vector $\mathbf{x}$. $\|\mathbf{X}\|_{F}$ denotes the Frobenius norm of matrix $\mathbf{X}$ and $\mathcal{N}_{\mathscr{C}}\left(0, \sigma^{2}\right)$ denotes a circular symmetric complex Gaussian distribution with zero mean and variance $\sigma^{2}$.

\section{System Model and Eigenmode Representation}

Let us consider a MIMO system with $n_{T}$ transmit antennas and $n_{R}$ receive antennas; the received signal is therefore given by

$$
\mathbf{y}=\mathrm{GAFs}+\mathrm{Gn},
$$

where $\mathbf{y}$ is the $b \times 1$ received symbol vector, $\mathbf{A}$ is the $n_{R} \times n_{T}$ channel matrix, $\mathbf{F}$ is the $n_{T} \times b$ linear precoding matrix, $\mathbf{G}$ is the $b \times n_{R}$ linear decoding matrix, $s$ is the $b \times 1$ transmitted symbol vector, and $\mathbf{n}$ is $n_{R} \times 1$ complex additive white Gaussian noise vector with zero mean and covariance matrix $N_{0} \mathbf{I}_{n_{R}}$. Let us assume that

$$
E\left[\mathbf{s s}^{H}\right]=E_{s} \mathbf{I}_{b}, \quad E\left[\mathbf{s n}^{H}\right]=0, \quad E\left[\mathbf{n} \mathbf{n}^{H}\right]=N_{0} \mathbf{I}_{n_{R}} .
$$

The average transmitted power is limited; the precoding matrix is normalized and is therefore subject to the power constraint:

$$
\|\mathbf{F}\|_{F}^{2}=1
$$

All over the paper, the channel state information is assumed to be perfectly known at both transmitter and receiver sides. We apply a transformation in order to obtain a simplified model of the system. This will result in a diagonalized channel matrix [3]. Let us apply this virtual transformation, which is based on the singular value decomposition (SVD), by means of the following decompositions: $\mathbf{A}=\mathbf{U A}_{v} \mathbf{V}^{H}, \mathbf{F}=\mathbf{F}_{v} \mathbf{F}_{d}, \mathbf{G}=\mathbf{G}_{v}$, where $\mathbf{F}_{v}=\mathbf{V}(:, 1: b)$ and $\mathbf{G}_{v}=\mathbf{U}^{H}(:, 1: b)$.

Relation (1) can be then rewritten as

$$
\mathbf{y}=\mathbf{A}_{v} \mathbf{F}_{d} \mathbf{s}+\mathbf{n}_{v}
$$

where $\mathbf{A}_{v}=\mathbf{G}_{v} \mathbf{A} \mathbf{F}_{v}$ is the eigenchannel matrix, $\mathbf{n}_{v}=\mathbf{G}_{v} \mathbf{n}$ is the transformed additive noise vector with the covariance matrix $\mathbf{R}_{\mathbf{n}_{v}}=E\left[\mathbf{n}_{v} \mathbf{n}_{v}{ }^{H}\right]=N_{0} \mathbf{I}_{b}$, and the unitary matrices $\mathbf{G}_{v}$ and $\mathbf{F}_{v}$ are chosen so as to ensure the whitening of the noise, the diagonalization of the channel, and the reduction of the dimension to $b$. The eigen-channel matrix $\mathbf{A}_{v}$ can now be expressed as

$$
\mathbf{A}_{v}=\operatorname{diag}\left(\sigma_{1}, \sigma_{2}, \ldots, \sigma_{b}\right)
$$

where the singular values are sorted in descending order.

\section{Low Density Parity Check Codes}

Low density parity check (LDPC) codes were proposed by Gallager in 1962 [4]. They are defined by a sparse parity check matrix over a Galois field $\operatorname{GF}(q)$, where $q$ denotes the order of the Galois field. LDPC codes are binary when $q=2$ and nonbinary when $q>2$. Binary LDPC codes have been shown to approach Shannon limit performance [4-7] for very long code lengths. NB-LDPC codes are usually preferred to their binary counterparts when the block length is small to moderate [8] or when the order of the symbols sent through the channel is not binary [9], which is the case for high order modulations or for multiple-antennas channels $[10,11]$. Davey and MacKay studied in 1998 NB-LDPC codes [12] and showed that they may achieve superior performance than the binary codes when constructed over higher order Galois fields at the expense of increased decoding complexity $[12,13]$.

A sparse parity check matrix $\mathbf{H}$ describes the LDPC code and can be efficiently represented by a bipartite graph called Tanner graph. Iterative decoding of LDPC codes has been addressed efficiently in $[12,14]$ using the Belief Propagation (BP) algorithm.

The $\mathrm{BP}$ algorithm is a suboptimal decoding algorithm proposed by Gallager in [4] for binary LDPC codes decoding and then generalized in [12] for the NB-LDPC codes. The iterative decoding is done by repeating the steps of the algorithm until a valid codeword has been obtained or a fixed number of iterations have been completed. Therefore, the rapidity of convergence of the algorithm appears to be an important issue.

One advantage of NB-LDPC coding is that one can match the field order with the constellation size. This way, one element in $\mathrm{GF}(q)$ is mapped to one point in the signal constellation. This is because the likelihood probabilities (or LLRV) for each coded symbol over GF( $q$ ) are independent of other coded symbols.

The LDPC codes we chose to use have the following properties. 
(i) Ultrasparse NB-LDPC codes with column weight $d_{v}=2$ at the variable-node side that gives large girths compared to classical binary graphs $[8,15]$.

(ii) These codes are well designed for both good waterfall and error floor properties $[8,15]$, compared to random choice of the Tanner graph structure and the nonzero values assignment.

(iii) At the check mode side, several row weights are chosen $\left(d_{c}=4,8\right.$, and 12$)$ to have precoding comparison at several code rates.

(iv) These codes are targeted for LTE-A with small packet lengths (small to moderate codeword lengths) [15].

(v) Small packet lengths are typically well suited for closed-loop MIMO systems.

\section{Linear Precoding}

The full knowledge of the channel state information at the transmitter side (Tx-CSI) permits the application of linear precoding operation in MIMO transmissions. It consists in a matrix multiplication operation that is applied to the transmitted signal which combines the symbols on the different antennas. This antenna allocation operation is determined and designed in such a way that allows the optimization of a well-defined criterion. Therefore, different linear precoders are defined because of the different optimization criteria. These precoders are mainly divided into two categories: diagonal precoders that allow transforming the MIMO transmission system into parallel independent SISO systems and nondiagonal precoders.

The precoders are detailed hereafter for the sake of presenting them on the same formalism, which facilitates the presentation of our proposed scheme and allows the comparison in terms of both EXIT analysis (Section 6) and numerical simulations (Section 7).

4.1. Diagonal Precoders. For diagonal precoders, the precoding matrix has the following expression:

$$
\mathbf{F}_{d}=\operatorname{diag}\left(\sqrt{p_{1}}, \ldots, \sqrt{p_{b}}\right),
$$

where $p_{i}(i=1, \ldots, b)$ represents the power allocation of the $i$ th subchannel.

Knowing that the transformation of the channel model gives a diagonal channel $\mathbf{A}_{v}$ the use of a diagonal precoder will result in completely independent subchannels and the transmission system is therefore equivalent to distributing the symbols onto parallel independent subsystems.

The power constraint in this case is expressed as

$$
\sum_{i=1}^{b} p_{i}=1
$$

The main optimization criteria that lead to diagonal precoders are the maximization of the postprocessing SNR [16], the maximization of the channel capacity [17], the minimization of the BER [18], the minimization of the mean square error (MSE) [19], the maintaining of fixed ratios between the postprocessing SNR values of the different subchannels [19].

4.1.1. Max-SNR Precoder. Maximizing the postprocessing SNR leads to a transmission over one subchannel only, corresponding to the highest subchannel singular value $\sigma_{1}$. If we apply the power constraint, we obtain $p_{1}=1$ and $p_{i}=0$ for $i=2, \ldots, b$. The max-SNR solution simplifies the signal model equation that becomes

$$
y=\sigma_{1} s+n
$$

4.1.2. Water-Filling Precoder. The optimization criterion that maximizes the channel capacity leads to the Water-Filling (WF) precoder. The channel capacity can be expressed as

$$
C=\sum_{i=1}^{b} \log _{2}\left(1+\frac{E_{s}}{N_{0}} \times p_{i} \sigma_{i}^{2}\right)
$$

with $E_{s}=E\left[|s|^{2}\right]$ and $N_{0}=E\left[|n|^{2}\right]$.

We set

$$
\tilde{\sigma}_{i}=\sigma_{i} \sqrt{\frac{E_{s}}{N_{0}}}
$$

and then the maximization of $C$ gives the following power allocation strategy:

$$
\begin{array}{r}
p_{i}= \begin{cases}\Psi-\frac{1}{\widetilde{\sigma}_{i}^{2}} & \text { if } \Psi>\frac{1}{\widetilde{\sigma}_{i}^{2}} \\
0 & \text { otherwise }\end{cases} \\
\text { for } i=1, \ldots, b,
\end{array}
$$

where the threshold $\Psi$ is defined by

$$
\begin{gathered}
\Psi=\frac{1+\gamma_{\Psi}}{b_{\Psi}} ; \\
\gamma_{\Psi}=\sum_{i=1}^{b_{\Psi}} \frac{1}{\widetilde{\sigma}_{i}^{2}}
\end{gathered}
$$

and $b_{\Psi}=i$ the greatest value in $\{1, \ldots, b\}$ such that $\Psi>1 / \widetilde{\sigma}_{i}^{2}$.

The power allocation strategy of the WF precoder depends on the SNR values of the subchannels (i.e., $\widetilde{\sigma}_{i}^{2}$ for $i=1,2, \ldots, b)$. The precoder allows the cancellation of the most disadvantaged subchannels. The total average power is then distributed among the strongest $b_{\Psi}$ subchannels.

In a $(2,2)$ MIMO system, this results in either transmitting over 2 subchannels or using only the most advantageous one. We will show later the switch of this precoder in function of the SNR values.

4.1.3. MMSE Precoder. The optimization criterion of the MMSE precoder is the minimization of the mean square error that is expressed as

$$
\operatorname{MSE}=E\left[\|\mathbf{y}-\mathbf{s}\|^{2}\right]=\sum_{i=1}^{b} E\left[\left|\left(\sigma_{i} \sqrt{p_{i}}-1\right) s_{i}+n_{i}\right|^{2}\right]
$$


and then the minimization of MSE gives the following power allocation strategy:

$$
p_{i}= \begin{cases}\frac{1}{\widetilde{\sigma}_{i}}\left(\Psi_{\text {MMSE }}-\frac{1}{\widetilde{\sigma}_{i}}\right) & \text { if } \Psi_{\text {MMSE }}>\frac{1}{\widetilde{\sigma}_{i}} \\ \text { otherwise } \\ \text { for } i=1, \ldots, b,\end{cases}
$$

where the threshold $\Psi_{\text {MMSE }}$ is defined by

$$
\begin{gathered}
\Psi_{\text {MMSE }}=\frac{1+\gamma_{\Psi_{\text {MMSE }}}}{\sum_{i=1}^{b_{\Psi_{M M S E}}}\left(1 / \widetilde{\sigma}_{i}\right)} ; \\
\gamma_{\Psi_{\text {MMSE }}}=\sum_{i=1}^{b_{\Psi_{\text {MMSE }}} \frac{1}{\widetilde{\sigma}_{i}^{2}} .}
\end{gathered}
$$

The power allocation strategy of the MMSE precoder depends on the SNR values of the subchannels also and may lead to subchannel cancellation; that is, $b_{\Psi_{\text {MMSE }}}$ subchannels are used (in the case of $b=2$, only one subchannel is used).

4.1.4. Quality of Service and Equal Error Precoders. The power allocation strategy of the Quality of Service (QoS) precoder aims to maintain fixed ratios between the postprocessing SNR values of the different subchannels [19], which are expressed as

$$
\gamma_{i}=p_{i} \widetilde{\sigma}_{i}^{2}=\frac{w_{i}}{\sum_{k=1}^{b} w_{k} / \widetilde{\sigma}_{k}^{2}} \quad \text { for } i=1, \ldots, b,
$$

where $w_{1}=1>w_{2}>\cdots>w_{b}$ are the fixed SNR ratios between subchannel $i \neq 1$ and subchannel 1 (i.e., $w_{i}=\gamma_{i} / \gamma_{1}$ ).

The Equal Error (EE) precoder is the particular case of the QoS precoder where all subchannels have the same postprocessing SNR value $\gamma_{i}$ by setting $w_{1}=w_{2}=\cdots=w_{b}=$ 1.

4.2. Nondiagonal Precoders. Unlike diagonal precoders, the precoding matrix of nondiagonal precoders is a nondiagonal matrix. In our study, we focus on two of the best performing nondiagonal precoders which are based on the minimal Euclidean distance criterion: the max- $d_{\min }$ precoder [3] and the max- $d_{\min }$-DFT precoder (Discrete Fourier Transform) [20].

4.2.1. Max $-d_{\min }$. The minimum Euclidean distance between signal points on the receiver's constellation affects the system performances, especially with the ML detector [21]. The max$d_{\text {min }}$ precoder is designed with the criterion of maximizing this minimal Euclidean distance $d_{\min }$ which is expressed as

$$
d_{\min }=\min _{\boldsymbol{\epsilon} \in \mathscr{E}}\left\|\mathbf{A}_{v} \mathbf{F}_{d} \boldsymbol{\epsilon}\right\|,
$$

where $\boldsymbol{\epsilon}=\mathbf{s}_{k}-\mathbf{s}_{l}$ is the error vector between vector symbols $\mathrm{s}_{k}$ and $\mathrm{s}_{l}$ for $k \neq l, \mathscr{E}$ being the set of all error vectors. The max $-d_{\min }$ precoder can then be obtained by solving

$$
\mathbf{F}_{d}^{d_{\min }}=\underset{\mathbf{F}_{d}}{\operatorname{argmax}} d_{\min }\left(\mathbf{F}_{d}\right)
$$

under the power constraint $\left\|\mathbf{F}_{d}\right\|_{F}^{2}=1$.
Collin et al. give in [3] a solution for (17) for the case of $b=2$ and a 4-QAM modulation. For two data streams, the precoding matrix is written as

$$
\mathbf{F}_{d}=\left(\begin{array}{cc}
\cos \psi & 0 \\
0 & \sin \psi
\end{array}\right)\left(\begin{array}{cc}
\cos \theta & \sin \theta \\
-\sin \theta & \cos \theta
\end{array}\right)\left(\begin{array}{cc}
1 & 0 \\
0 & e^{j \phi}
\end{array}\right)
$$

and the channel matrix is written as

$$
\mathbf{A}_{v}=\rho\left(\begin{array}{cc}
\cos \gamma & 0 \\
0 & \sin \gamma
\end{array}\right)
$$

where $\rho^{2}=\sigma_{1}^{2}+\sigma_{2}^{2}$ and $\tan \gamma=\sigma_{2} / \sigma_{1}$.

For 4-QAM modulation, the values of the triplet $(\psi, \theta, \phi)$ that maximizes $d_{\text {min }}$ depend on the parameter $\gamma$, and two cases are possible:

(i) if $\gamma<\gamma_{0}$, we have

$$
\begin{aligned}
& \psi=0, \\
& \theta=\operatorname{atan}\left(\frac{2}{(\sqrt{6}+\sqrt{2})}\right), \\
& \phi=15^{\circ} ;
\end{aligned}
$$

(ii) if $\gamma \geq \gamma_{0}$, we have

$$
\begin{aligned}
& \psi=\operatorname{atan}\left(\frac{(\sqrt{2}-1)}{\tan \gamma}\right), \\
& \theta=45^{\circ}, \\
& \phi=45^{\circ}
\end{aligned}
$$

with $\gamma_{0} \approx 17.28^{\circ}$.

4.2.2. $M a x-d_{\min }-D F T$. The general form of this precoder can be written as

$$
\mathbf{F}_{d}=\operatorname{diag}\left(\sqrt{p_{1}}, \ldots, \sqrt{p_{b}}\right) W_{b},
$$

where $W_{b}$ is the DFT matrix defined as

$$
W_{b}=\left(w^{k l}\right)_{k, l=0, \ldots, b-1}
$$

with $w=e^{j 2 \pi / b}$.

For $b=2$, the DFT matrix is written as

$$
W_{2}=\left(\begin{array}{cc}
1 & 1 \\
1 & -1
\end{array}\right) \text {. }
$$

The power allocation strategy aims to optimize the minimal Euclidean distance $d_{\min }$, which leads to a switch to the max-SNR solution at $\gamma_{\mathrm{DFT}}=\operatorname{atan}(1 / \sqrt{7})=20.70^{\circ}$. 


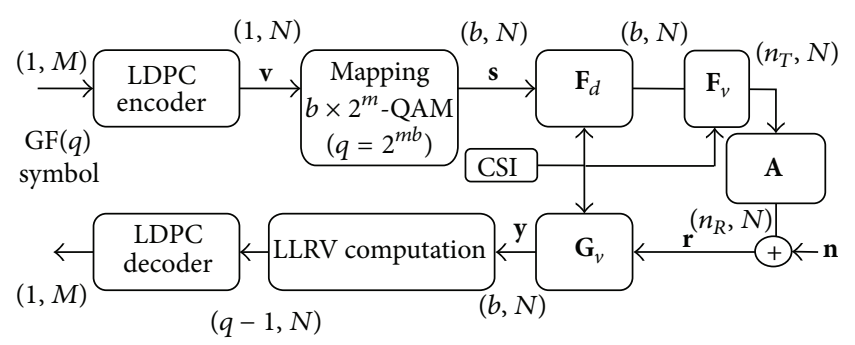

FIGURE 1: Proposed block diagram scheme.

For $\gamma<\gamma_{\mathrm{DFT}}$, the max-SNR precoder is used, while for $\gamma>\gamma_{\mathrm{DFT}}$, considering the form expressed in (19), we obtain the following triplet values:

$$
\begin{aligned}
& \psi=\operatorname{atan}\left(\frac{1}{(\sqrt{3} \tan \gamma)}\right), \\
& \theta=45^{\circ} \\
& \phi=0 .
\end{aligned}
$$

\section{Proposed Scheme}

The block diagram of the proposed scheme is detailed in Figure 1. A sparse parity check matrix $\mathbf{H}$ of dimension $(M \times$ $N)$ whose elements are in the Galois field $\mathrm{GF}(q)$ defines the LDPC code, with $M=N-K$, where $K$ and $N$ are, respectively, the source block length and the transmitted code block length. Data stream is encoded through the LDPC encoder into the codeword $\mathbf{v}$ and then mapped into $b \times 2^{m}$-QAM symbol vectors. Note that the code alphabet matches the $b$-dimensional constellation alphabet (i.e., $q=$ $2^{m b}$ ). The precoding matrix $\mathbf{F}_{d}$ is applied to the transmitted symbol vectors before transmission through the channel $\mathbf{A}_{v}\left(\mathbf{A}_{v}=\mathbf{G}_{v} \mathbf{A F}_{v}\right)$ where the transformed AWGN noise vector is added. The received symbol vector $\mathbf{y}$ is used to compute the log likelihood ratio vector (LLRV) required for log-BP initialization.

The LLRV corresponding to the variable-node $v$, according to the channel model, is given by

$$
\mathbf{L}_{\mathbf{v}}=\left[\begin{array}{lllll}
L_{v}(v=1) & L_{v}(v=\alpha) & \cdots & L_{v}\left(v=\alpha^{q-2}\right)
\end{array}\right],
$$

where $\left\{0,1, \alpha, \ldots, \alpha^{q-2}\right\}$ are the elements of $\mathrm{GF}(q), \alpha$ represents the primitive element, and

$$
L_{v}\left(v=\alpha^{k}\right)=\log \left(\frac{P\left(\mathbf{y} \mid v=\alpha^{k}\right)}{P(\mathbf{y} \mid v=0)}\right) \quad \text { for } k=0, \ldots, q-2 .
$$

The channel LLR value computation is actually done using the following expression:

$$
L_{v}\left(v=\alpha^{k}\right)=-\frac{1}{N_{0}}\left(\left\|\mathbf{y}-\mathbf{A}_{v} \mathbf{F}_{d} \mathbf{s}^{(k+1)}\right\|^{2}-\left\|\mathbf{y}-\mathbf{A}_{v} \mathbf{F}_{d} \mathbf{s}^{(0)}\right\|^{2}\right),
$$

where $\mathbf{s}^{(k)}$ represent all possible elements of vectors $\mathbf{s}$ for $k=$ $0,1, \ldots, q-2$.

The LLRV values of (28), calculated for all nonbinary GF symbols of a codeword, are the intrinsic messages given as input to the Belief Propagation algorithm. They serve to initialize extrinsic messages, which are then updated through the iterated exchange of messages between variable and check nodes in the Tanner graph representing the code. At the end of every iteration, a posteriori messages are calculated for a decoding attempt. The procedure stops when all the parity check equations are satisfied (null syndrome) or when the maximum number of iterations is reached (decoding failure).

\section{EXIT Analysis}

EXIT charts predict the behavior of the BP decoder on the basis of only simulated behavior of the individual components decoders (variable-node decoder (VND) and checknode decoder (CND)). Using this approach it is only necessary to simulate the behavior of each component decoder once. Such charts that track the mutual information at each iteration give an excellent visual representation to analyze the decoding convergence.

In [9], Bennatan and Burshtein extend the development of EXIT charts for binary LDPC codes to NB-LDPC codes. In the following, the main results are presented.

The mutual information $I(C ; \mathbf{L})$ between the code symbol $C$ and its corresponding LLR vector $\mathbf{L}$ (a priori or extrinsic information from VND and CND at each iteration step) can be expressed as

$$
I(C ; \mathbf{L})=1-E\left[\log _{q}\left(1+\sum_{i=1}^{q-1} e^{-\mathbf{L}_{\mathbf{i}}}\right) \mid C=0\right] .
$$

The conditioning on $C=0$ results in the classical allzero codeword assumption where the decoder performance is independent of the transmitted codeword.

Using a Gaussian distribution approximation of $\mathbf{L}$ (mean $\mathbf{m}$ and covariance matrix $\Sigma$ ) and symmetry and permutation invariance assumptions as defined in [9], the number of parameters is reduced from $q-1$ to one parameter denoted by $\sigma$ such that

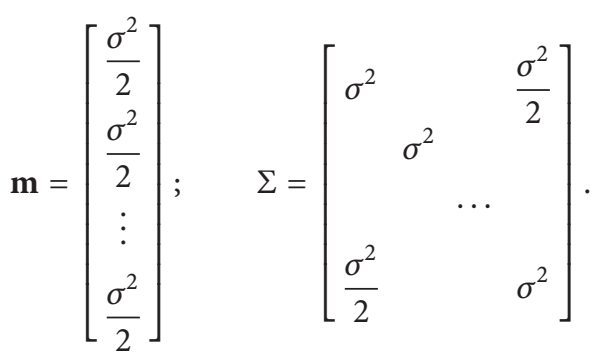

That is, $m_{i}=\sigma^{2} / 2, i=1, \ldots, q-1$, and $\Sigma_{i, j}=\sigma^{2}$ if $i=j$ and $\sigma^{2} / 2$ otherwise.

The computation of (30) can be numerically evaluated by using the Gaussian approximation (31) and results in a monotonically increasing function $J_{q}(\sigma)=I(C ; \mathbf{L})$ with values from 0 to 1 . We denote by $J_{q}^{-1}(I)$ the corresponding 
inverse function. These functions can be obtained by using fitting polynomial approximation [9].

These assumptions result in the expression of the classical mutual information relations for the CND and VND regular LDPC codes, similarly to the binary case:

$$
\begin{aligned}
& I_{E, \mathrm{VND}}\left(I_{A} ; d_{v}, I^{(0)}\right) \\
& \quad \approx J_{q}\left(\sqrt{\left(d_{v}-1\right)\left[J_{q}^{-1}\left(I_{A}\right)\right]^{2}+\left[J_{q}^{-1}\left(I^{(0)}\right)\right]^{2}}\right), \\
& I_{E, \mathrm{CND}}\left(I_{A} ; d_{c}\right) \approx 1-J_{q}\left(\sqrt{d_{c}-1} J_{q}^{-1}\left(1-I_{A}\right)\right),
\end{aligned}
$$

where $I_{A}$ and $I^{(0)}$ denote the mutual information of the incoming and the initial message, respectively. $I^{(0)}$ equals the capacity of the channel and is given by

$$
I^{(0)}=1-\frac{1}{q} \sum_{i=1}^{q} E_{\mathbf{n}_{v}}\left\{\log _{q} \sum_{j=1}^{q} e^{-d_{i, j}}\right\}
$$

with $d_{i, j}=\left(1 / N_{0}\right)\left(\left\|\mathbf{A}_{v} \mathbf{F}_{d}\left(\mathbf{s}_{i}-\mathbf{s}_{j}\right)+\mathbf{n}_{v}\right\|^{2}-\left\|\mathbf{n}_{v}\right\|^{2}\right)$ and $q=2^{m b}$.

Unlike (33), (32) depends on the channel information (SNR value and MIMO precoding). Based on (32) the computation of $I_{E, \mathrm{VND}}$ highly depends on the mutual information $I^{(0)}$. Higher $I^{(0)}$ implies a higher $I_{E, \mathrm{VND}}$ curve $\left(J_{q}(\sigma)\right.$ and $J_{q}^{-1}(I)$ monotonically increasing functions), which means that the convergence point with the $I_{E, \mathrm{CND}}$ curve will be closer to one. Therefore, by examining the $I^{(0)}$ curves for the different precoders, we should be able to predict the performance of the association with the LDPC code.

In Figure 2, the mutual information $I^{(0)}$ is plotted for every precoder for $b=2$ data streams in function of $\gamma$ (cf. (20) and (34)). This plot is shown for a given received normalized SNR equal to $8.0 \mathrm{~dB}$ and defined as

$$
\mathrm{SNR}_{R}=\frac{\|\mathbf{A}\|_{F}^{2}}{n_{R} n_{T}} \frac{E_{s}}{N_{0}} .
$$

Since the channel energy $\|\mathbf{A}\|_{F}^{2}=\rho^{2}$ is included in $\mathrm{SNR}_{R}$, $I^{(0)}$ does not depend on $\rho^{2}$. Thus $I^{(0)}$ shows only the influence of the parameter $\gamma$ for each precoder (cf. (20) and (34)). Note that the values of $I^{(0)}$ are between 0 and 1 since logarithm $\log _{q}$ uses base $q=2^{m b}$. In terms of bit per symbol time, the channel capacity is equal to $b m I^{(0)}$, with $b=2$, and $m=2$ (4-QAM); values are between 0 and $b m=4$.

In order to maintain a constant transmission throughput and knowing that some precoders may cancel the weak subchannel and transmit only on the best one, the modulation of the data stream was set to switch to 16-QAM whenever the precoder cancels one subchannel. Therefore the precoders will be denoted in the simulation results as presented in Table 1.

In order to analyze the performance of the precoders in a given Rayleigh MIMO system, it is important to examine the distribution (pdf) of the $\gamma$ angle. Therefore, Figure 3 plots the probability density function of $\gamma$ for Rayleigh channel model
TABLE 1: Precoder notations.

\begin{tabular}{lc}
\hline Precoder & Notation \\
\hline Max-SNR $(1 \times 16$ QAM $)$ & MaxSNR \\
Water-Filling $(2 \times 4$ QAM $/ 1 \times 16$ QAM $)$ & WF \\
MMSE $(2 \times 4$ QAM/1 $\times 16$ QAM $)$ & MMSE \\
Equal Error $(2 \times 4$ QAM $)$ & EE \\
Quality of Service & QoS \\
$\left(2 \times 4\right.$ QAM-WdB with $\left.W=10 \log _{10} w_{2}\right)$ & MaxDmin \\
Max- $d_{\text {min }}(2 \times 4$ QAM $)$ & MaxdminDFT \\
Max- $d_{\text {min }}-$ DFT- $2 \times 4$ QAM $/ 1 \times 16$ QAM &
\end{tabular}

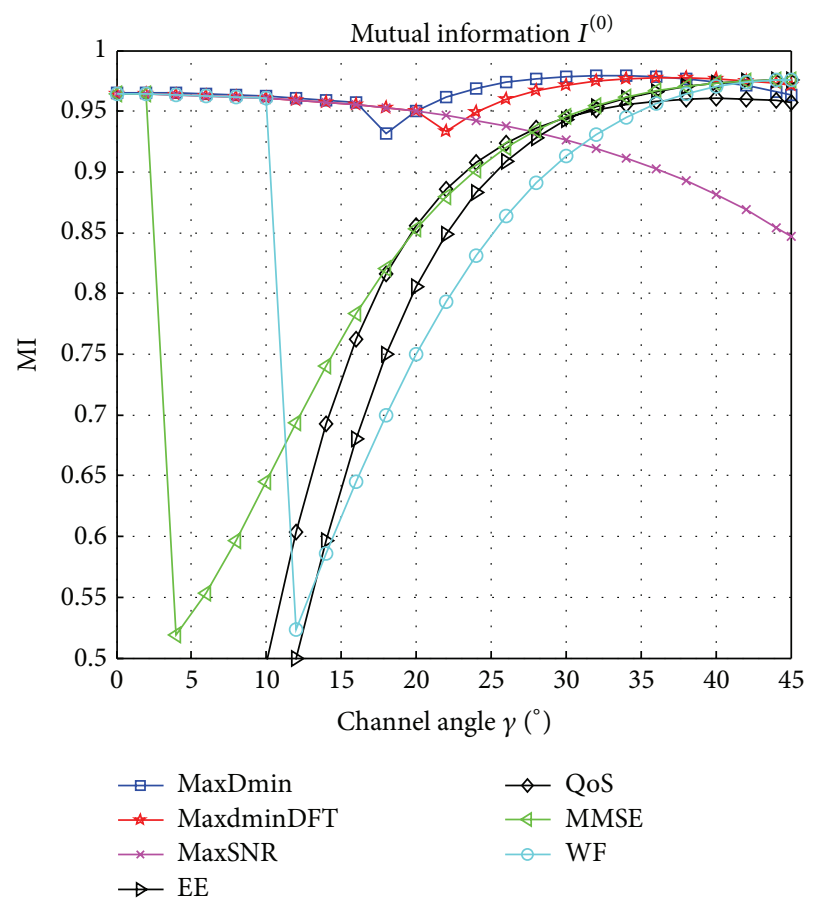

FIGURE 2: Mutual information $I^{(0)}$ in function of $\gamma$ for different precoders.

$\left([\mathrm{A}]^{i j} \sim \mathcal{N}_{\mathscr{C}}(0,1)\right)$ for both $\operatorname{MiMO}(2,2)$ and $\operatorname{MiMO}(2,4)$ systems [22].

Figure 3 shows that, in the case of $\operatorname{MIMO}(2,2)$ system, the pdf is larger for low $\gamma$ values than for high $\gamma$ values, while, in the case of MIMO $(2,4)$ system, the pdf is considerably larger for high $\gamma$ values than for low $\gamma$ values. This will be helpful in order to explain the BER performance with regard to the $I^{(0)}$ values in function of $\gamma$. The $I^{(0)}$ values for low $\gamma$ values will affect the $\operatorname{MIMO}(2,2)$ system and the $I^{(0)}$ values for high $\gamma$ values will affect the $\operatorname{MIMO}(2,4)$ system performance.

Now that $\gamma$ distribution is known for both MIMO systems, the impact of $\gamma$ on $I^{(0)}$ for every precoder can be analyzed, and the performance of each precoder can be predicted for both MIMO systems.

In Figure 2, the curve corresponding to the Equal Error precoder has the lowest values for small $\gamma$ values and does not increase much for large $\gamma$ values, compared to other 


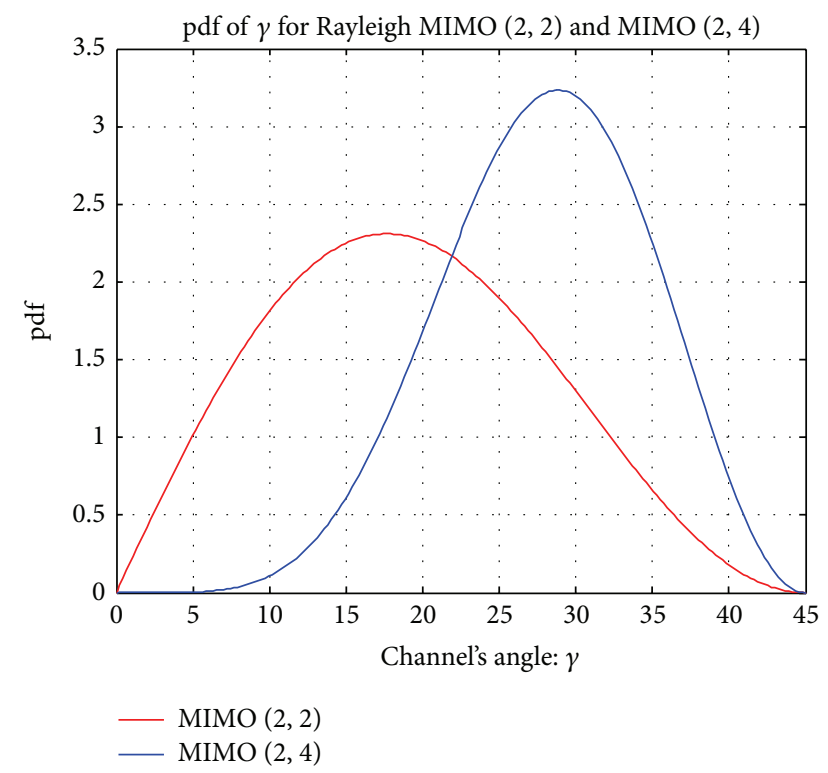

FIgURE 3: Probability density functions of the $\gamma$ channel angle for Rayleigh MIMO $(2,2)$ and MIMO $(2,4)$ systems.

precoders. This will affect the performance of this precoder that should be among the worse compared to other precoders especially in the case of $\operatorname{MIMO}(2,2)$ system.

The curve of the QoS precoder is slightly higher than the EE one, which will result in a slight performance improvement for both MIMO systems.

The WF precoder is a diagonal precoder that is capable of using two subchannels or cancelling the second when the threshold stated in (11) is exceeded. This appears in its curve where high $I^{(0)}$ values are recorded for small $\gamma$ values, and a switch is clearly seen above a $\gamma$ limit, where this precoder becomes even worse than the EE. This deterioration will be retrieved in the performance level when $\gamma$ values increase (MIMO $(2,4))$.

The MMSE precoder applies a similar cancellation strategy as the WF with a smaller switch angle. Its curve shows lower $I^{(0)}$ values than the WF for small $\gamma$ and greater $I^{(0)}$ values for large $\gamma$. This will be reflected in better performance of MMSE compared to WF in the case of MIMO $(2,4)$ system.

These curves show that all diagonal precoders have low $I^{(0)}$ values, which predicts poor performance in terms of BER.

The max-SNR precoder, which can be considered as a special precoder because of its use of one subchannel only, reaches high $I^{(0)}$ values for small $\gamma$ values. For high $\gamma$ values, the precoder strategy is to always use one subchannel only, thus wasting the second subchannel that became more advantageous when $\gamma$ values are high. The max-SNR $I^{(0)}$ curve decreases with increasing $\gamma$ and becomes low. The maxSNR precoder will have performance as good as nondiagonal precoders for MIMO $(2,2)$ system and will see its performance deteriorated for MIMO $(2,4)$ system.

Finally, the nondiagonal precoders based on the minimum Euclidean distance criterion $\left(\max -d_{\min }\right.$ and $\max -d_{\min }{ }^{-}$ DFT) have both a constant $I^{(0)}$ value larger than all the

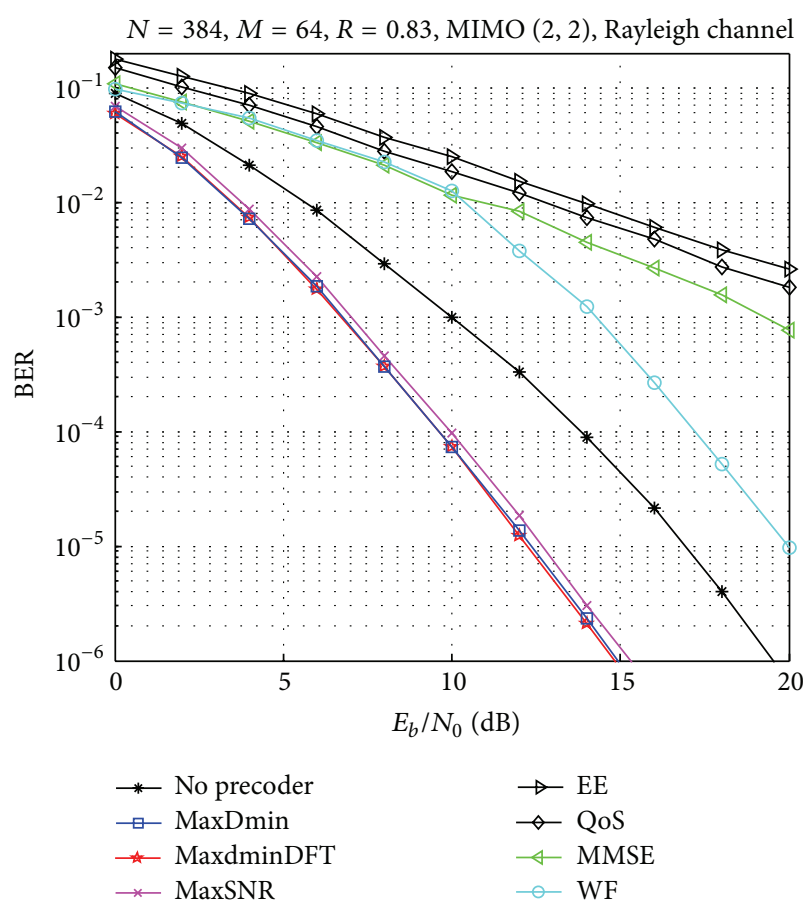

FIGURE 4: BER performance of the proposed scheme for a $(2,2)$ MIMO system.

others, whatever the $\gamma$ values are. This will result in a better performance of both MIMO systems.

As it is shown in (34), the minimum Euclidean distance appears in the computation of $I^{(0)}$, and the curves of Figure 2 confirm that this criterion is well-suited with the LDPC code association.

In addition, the high values of $I^{(0)}$ for the nondiagonal precoders predict not only better performance in terms of BER, but also a higher convergence rate due to the smaller number of iterations needed in order to reach convergence point on the EXIT charts.

This analysis of the $I^{(0)}$ curves for different precoders will be verified through simulations and the predicted results will be confirmed hereafter (Figures 4 and 5).

\section{Simulation Results}

The performance of the proposed power allocation scheme has been validated through a series of simulations that assess the achieved improvement in terms of both BER and convergence rate.

The precoders under evaluation are all the above cited diagonal and nondiagonal precoders. LDPC codes are constructed over GF(16) and different parity check matrices are used, having different rates, all derived from matrices designed in the framework of DAVINCI Project $[8,23]$. Simulations have been run on two different MIMO configurations: a symmetric $(2,2)$ system and an asymmetric $(2,4)$ system. The number of subchannels is set to $b=2$. The modulation is set to 4-QAM. We consider a flat-fading channel with a Rayleigh distribution model. A new channel realization 


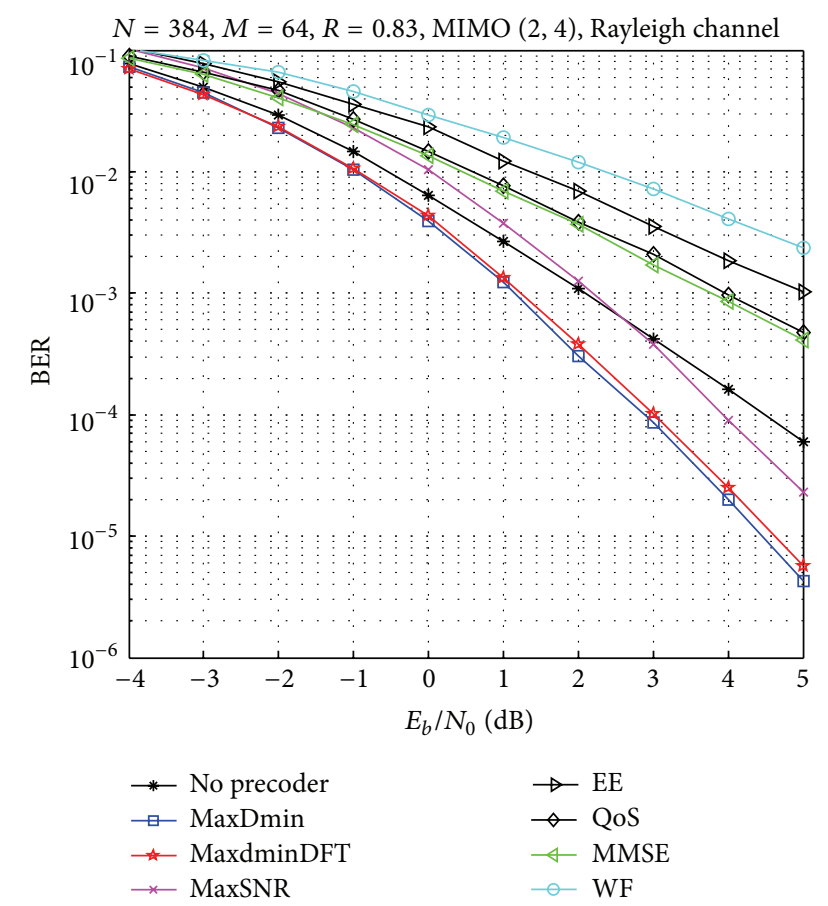

FIGURE 5: BER performance of the proposed scheme for a $(2,4)$ MIMO system.

TABLE 2: SNR per bit definition.

\begin{tabular}{lc}
\hline System with precoding & $E_{b} / N_{0}=\left(E_{s} / N_{0}\right)(1 / 4 R)$ \\
\hline $\begin{array}{l}\text { System without precoding } \\
\text { (spatial multiplexing) }\end{array}$ & $E_{b} / N_{0}=\left(n_{T} E_{s} / N_{0}\right)(1 / 4 R)$ \\
\hline
\end{tabular}

is considered at each transmitted codeword with $[\mathbf{A}]^{i j} \sim$ $\mathcal{N}_{\mathscr{C}}(0,1)$. The SNR per bit is defined in Table 2 .

Figure 4 illustrates the BER performances versus SNR for a $(2,2)$ MIMO transmission system with LDPC code designed from the parity check matrix having the following parameters:

(i) matrix size: $N=384, M=64$, the numbers of nonzero elements in columns and rows being, respectively, $d_{v}=2$ and $d_{c}=12$,

code rate: $R=5 / 6=0.83$,

girth: 8 .

The curves on this figure assess the performance of the proposed scheme predicted above by EXIT analysis and confirm the results for the MIMO $(2,2)$ system.

Figure 4 shows that applying diagonal precoders on MIMO systems with LDPC codes deteriorates the performance of the transmission. All diagonal precoders present higher BER than the system with no precoding that was plotted as reference. The only precoders that permit a gain of about $4 \mathrm{~dB}$ at high $E_{b} / N_{0}$ are the one based on the minimal Euclidean distance criteria, along with the max-SNR precoder.

Figure 5 illustrates another simulation done with the same configuration over an asymmetric $(2,4)$ MIMO system.
The results show that the diagonal precoders are still damaging the performance and that the $\max -d_{\min }$ and the max$d_{\min }$-DFT still allow a gain of up to $1 \mathrm{~dB}$. The max-SNR precoder shows a weaker performance than that of the symmetric $(2,2)$ MIMO system.

These simulations confirm the performance predicted from the $I^{(0)}$ values in Figure 2.

These results suggest that the power allocation optimization of LDPC coded MIMO systems should be done using minimal Euclidean distance based precoders.

The use of SVD allows transforming the MIMO channel matrix into $b$ parallel independent eigensubchannels. The diagonal precoder ( $\mathbf{F}_{d}$ is a diagonal matrix) preserves the diagonal structure, which allows transmitting each symbol over each eigensubchannel. The diagonal matrix $\mathbf{F}_{d}$ allows only obtaining a power allocation strategy among all the subchannels used. In this particular case, the diagonal structure leads to diversity loss. The diversity order of diagonal precoders using $b$ substreams is $\left(n_{T}-b+1\right)\left(n_{R}-b+1\right)$. Hence, increasing the throughput by sending multiple symbols at a time loses the full diversity order over flat-fading channel $[24,25]$. We obtain performance degradation in terms of BER. Only the special case for max-SNR precoder $(b=1)$ can reach full diversity order. The proposed NB-LDPC codes are not well suited for diagonal closed-loop systems $(b>1)$ due to the bad LLRV initialization of the log-BP algorithm. This bad initialization was clearly examined in the VND curves of the diagonal precoders (Figure 2). The max- $d_{\text {min }}$ precoder that presents full diversity order [22] is well suited with the proposed NB-LDPC. The $I^{(0)}$ values of Figure 2 have already predicted this good match.

Therefore, other simulations have been conducted on the max- $d_{\min }$ precoder (in which performances appear to be equivalent to the max- $d_{\min }$-DFT) in order to validate these results, and four different systems have been compared. The first one is a $(2,2)$ MIMO transmission without any error correcting coding or precoding. This system is equivalent to "spatial multiplexing (SM)." The second system consists in the same MIMO scheme with the LDPC coding block, which is referred to as "SM-LDPC." The third system consists in a MIMO scheme where the max- $d_{\min }$ precoder is applied and is referred to as "max- $d_{\text {min }}$ " The last system is the MIMO scheme with LDPC coding and where the power allocation optimization is done by applying the max $-d_{\min }$ precoder and is referred to as "max- $d_{\min }$-LDPC." Figures 6, 7, and 8 show BER performance of these four systems for LDPC codes rates of $0.5\left(d_{c}=4\right), 0.75\left(d_{c}=8\right)$, and $0.83\left(d_{c}=12\right)$, respectively.

In Figure 6 we can see that the SM-LDPC system reaches the BER value of $10^{-5}$ for $E_{b} / N_{0}$ equal to $12.5 \mathrm{~dB}$ while the max- $d_{\min }$-LDPC system reaches the same BER value for only $11 \mathrm{~dB}$. Consequently, the max- $d_{\min }-\mathrm{LDPC}$ system has a gain of $1.5 \mathrm{~dB}$ over the SM-LDPC system for this same BER value.

In Figure 7, it can be noted that, for a higher code rate, the gain of the max- $d_{\min }$-LDPC system over the SM-LDPC is increased and attains $4.2 \mathrm{~dB}$ for a BER value of $10^{-5}$.

In Figure 8, the increase of the gain between the max$d_{\text {min }}$-LDPC and the SM-LDPC systems reaches $5 \mathrm{~dB}$ for a BER value of $10^{-5}$ and confirms that the improvement 


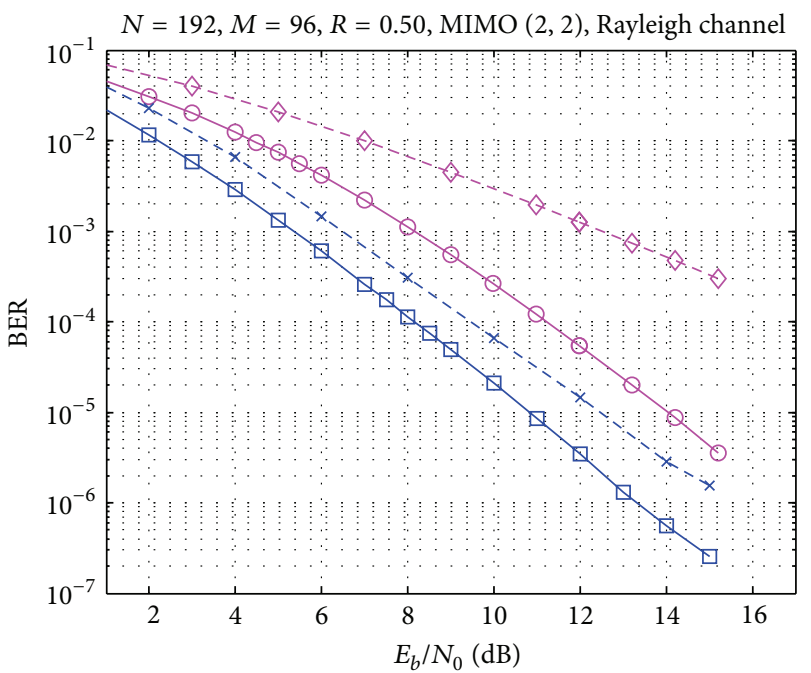

$$
\begin{array}{ll}
\square \text { Max- } d_{\min }-\text { LDPC } & -*-\text { SM-LDPC } \\
- \text { Max- } d_{\min } & -\diamond-\mathrm{SM}
\end{array}
$$

FIGURE 6: BER performance for max- $d_{\min }$ with LDPC code rate $R=$ $0.5\left(d_{c}=4\right)$.

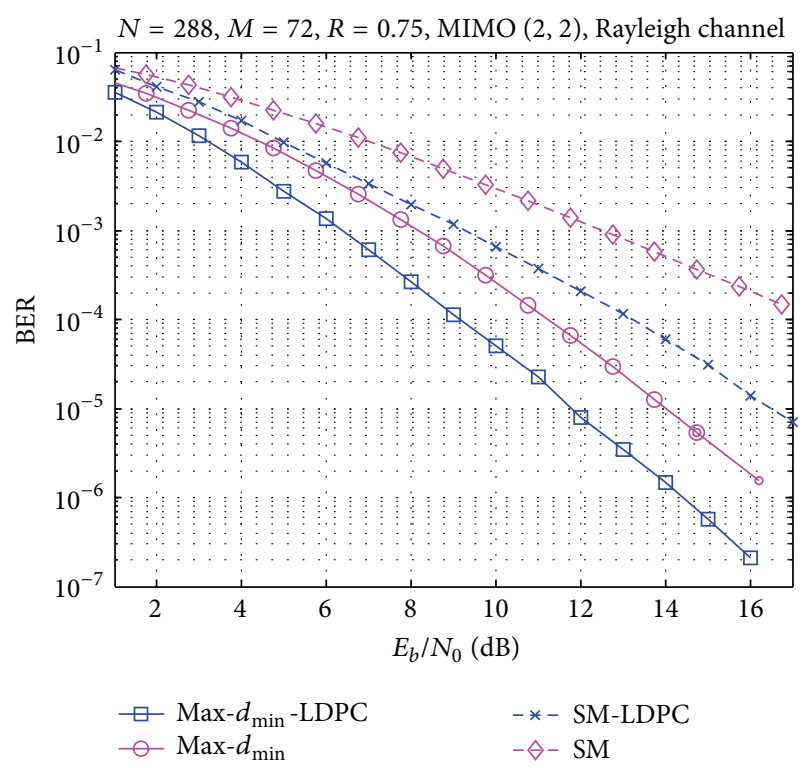

FIGURE 7: BER performance for max- $d_{\min }$ with LDPC code rate $R=$ $0.75\left(d_{c}=8\right)$.

brought by the max $-d_{\text {min }}$ precoder to an LDPC coded MIMO system is more significant for LDPC codes with higher rates.

The presence of the max- $d_{\text {min }}$ precoder allows an increase of the spectral efficiency compared to the SM-LDPC system. For example, we can see that the BER performance of the SM-LDPC for code rate $R=0.5$ (Figure 6) and the BER performance of the max- $d_{\text {min }}$-LDPC for code rate $R=0.83$ (Figure 8 ) are very similar. The transmission rate is $4 \times 0.5=2$ for SM-LDPC and $4 \times 0.83=3.33$ for $\max -d_{\min }$-LDPC. This is equivalent to a $67 \%$ increase in the transmission rate for the same BER performance, due to the use of the max- $d_{\min }$ precoder.

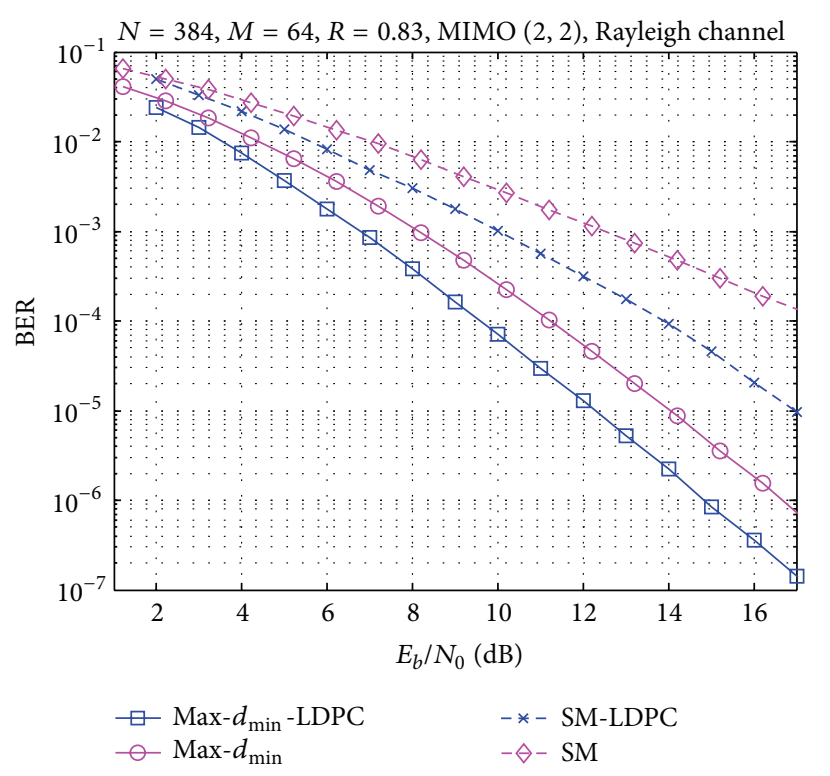

FIGURE 8: BER performance for $\max -d_{\min }$ with LDPC code rate $R=$ $0.83\left(d_{c}=12\right)$.

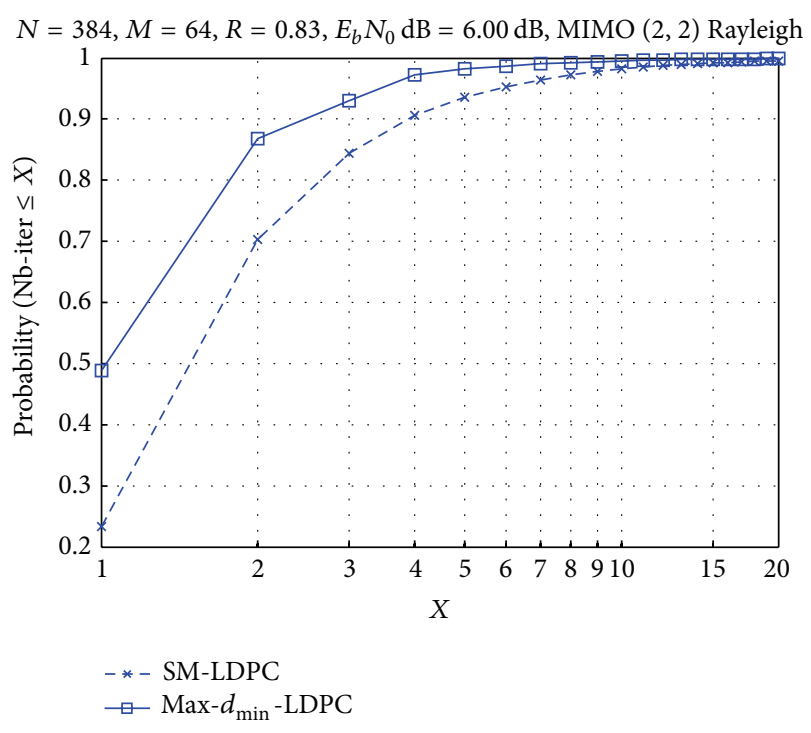

FIGURE 9: Probability of number of iterations till convergence for SM-LDPC and max- $d_{\min }$-LDPC transmissions.

The convergence rate of the LDPC decoder, when combined with linear precoding, has been appraised through the simulations and the results point out that a slight improvement is obtained compared to the SM-LDPC transmissions. This result has been already predicted and explained via the EXIT charts analysis above.

We denote by "Nb-iter" the required number of iterations for the BP algorithm to converge (i.e., null syndrome). Figure 9 plots the probability of $\mathrm{Nb}$-iter to be less than or equal to the numbers on the $x$-axis (abscissa), $P(\mathrm{Nb}$-iter $\leq X)$ for $X=1,2, \ldots, 20$ and for a fixed SNR per bit at $6 \mathrm{~dB}$. For $X=1$ the $\mathrm{BP}$ algorithm converges at the iteration number 1 in 
$23 \%$ of the cases for the SM-LDPC system, while it converges at the first iteration in $49 \%$ of the cases for the max- $d_{\min ^{-}}$ LDPC. For larger values of $X$, the gain becomes smaller, but still noticeable.

\section{Conclusion}

We have presented in this paper a new power allocation optimization scheme that deals with MIMO transmission scheme using ultrasparse NB-LDPC codes adapted for closed-loop MIMO systems and targeted for LTE-A with small to moderate codeword lengths. The proposed scheme makes use of a nondiagonal linear precoder based on the minimal Euclidean distance criteria in order to improve performance in terms of BER. Our study has shown that it is possible to join precoding techniques to such MIMO systems with NB-LDPC codes, but our simulation results also reveal that no improvement is brought by diagonal linear precoders when NB-LDPC codes are used. However, the special case of the max-SNR is competitive for $n_{T}=$ $n_{R}=2$ only. The results of our simulation are predicted and explained by a theoretical EXIT chart analysis that shows the impact of using different precoders on the initial mutual information of the decoder and subsequently its influence on the performance of the proposed association. We have established that using a full diversity order precoder (max$d_{\text {min }}$ or $\max -d_{\min }$-DFT) along with NB-LDPC results in a considerable improvement of the transmission performance and leads to a slight improvement of the convergence rate for the log Belief Propagation algorithm of LDPC decoder. Consequently, thanks to the proposed scheme, a smaller $\mathrm{SNR}$ is required to achieve a given BER value. The spectral efficiency is also increased due to the use of LDPC codes of higher rate along with the corresponding precoding. This result implies less energy consumption in the system and promotes green communication applications.

\section{Conflict of Interests}

The authors declare that there is no conflict of interests regarding the publication of this paper.

\section{Acknowledgments}

This work has been supported by the CORRIDOR (ANR) project and the Images \& Réseaux cluster.

\section{References}

[1] J. Wang, Z. Lv, and X. Li, "Analysis of MIMO diversity improvement using circular polarized antenna," International Journal of Antennas and Propagation, vol. 2014, Article ID 570923, 9 pages, 2014.

[2] R.-H. Peng and R.-R. Chen, "Design of non-binary LDPC codes over GF(q) for multiple-antenna transmission," in Proceedings of the Military Communications Conference, pp. 1-7, Washington, DC, USA, October 2006.

[3] L. Collin, O. Berder, P. Rostaing, and G. Burel, "Optimal minimum distance-based precoder for MIMO spatial multiplexing systems," IEEE Transactions on Signal Processing, vol. 52, no. 3, pp. 617-627, 2004.

[4] R. G. Gallager, "Low-density parity-check codes," IRE Transactions on Information Theory, pp. 21-28, 1962.

[5] T. J. Richardson, M. A. Shokrollahi, and R. L. Urbanke, "Design of capacity-approaching irregular low-density paritycheck codes," IEEE Transactions on Information Theory, vol. 47, no. 2, pp. 619-637, 2001.

[6] M. G. Luby, M. Mitzenmacher, M. A. Shokrollahi, and D. A. Spielman, "Improved low-density parity-check codes using irregular graphs," IEEE Transactions on Information Theory, vol. 47, no. 2, pp. 585-598, 2001.

[7] S.-Y. Chung, G. D. Forney Jr., T. J. Richardson, and R. L. Urbanke, "On the design of low-density parity-check codes within $0.0045 \mathrm{~dB}$ of the Shannon limit," IEEE Communications Letters, vol. 5, no. 2, pp. 58-60, 2001.

[8] C. Poulliat, M. Fossorier, and D. Declercq, "Design of regular $(2, \mathrm{dc})$-ldpc codes over $\mathrm{gf}(\mathrm{q})$ using their binary images," IEEE Transactions on Communications, vol. 56, no. 10, pp. 1626-1635, 2008.

[9] A. Bennatan and D. Burshtein, "Design and analysis of nonbinary LDPC codes for arbitrary discrete-memoryless channels," IEEE Transactions on Information Theory, vol. 52, no. 2, pp. 549$583,2006$.

[10] S. Pfletschinger and D. Declercq, "Non-binary coding for vector channels," in Proceedings of the IEEE 12th International Workshop on Signal Processing Advances in Wireless Communications (SPAWC '11), pp. 26-30, June 2011.

[11] A. Voicila, D. Declercq, F. Verdier, M. Fossorier, and P. Urard, "Low-complexity decoding for non-binary LDPC codes in high order fields," IEEE Transactions on Communications, vol. 58, no. 5, pp. 1365-1375, 2010.

[12] M. C. Davey and D. MacKay, "Low-density parity check codes over GF(q)," IEEE Communications Letters, vol. 2, no. 6, pp. 165$167,1998$.

[13] X.-Y. Hu and E. Eleftheriou, "Binary representation of cycle Tanner-graph GF(2b) codes," in Proceedings of the IEEE International Conference on Communications, pp. 528-532, Paris, France, June 2004.

[14] L. Barnault and D. Declercq, "Fast decoding algorithm for LDPC over GF $\left(2^{q}\right)$," in Proceedings of the IEEE Information Theory Workshop, pp. 70-73, Paris, France, March 2003.

[15] S. Pfletschinger, A. Mourad, E. Lopez, D. Declercq, and G. Bacci, "Performance evaluation of non-binary LDPC codes on wireless channels," in Proceedings of the ICT Mobile Summit Conference, 2009.

[16] P. Stoica and G. A. Ganesan, "Maximum-SNR spatial-temporal formatting designs for MIMO channels," IEEE Transactions on Signal Processing, vol. 50, no. 12, pp. 3036-3042, 2002.

[17] I. E. Telatar, "Capacity of multi-antenna Gaussian channels," European Transactions on Telecommunications, vol. 10, no. 6, pp. 585-595, 1999.

[18] P. Rostaing, O. Berder, G. Burel, and L. Collin, "Minimum BER diagonal precoder for MIMO digital transmissions," Signal Processing, vol. 82, no. 10, pp. 1477-1480, 2002.

[19] H. Sampath, P. Stoica, and A. J. Paulraj, "Generalized linear precoder and decoder design for MIMO channels using the weighted MMSE criterion," IEEE Transactions on Communications, vol. 49, no. 12, pp. 2198-2206, 2001. 
[20] Q.-T. Ngo, O. Berder, and P. Scalart, "General minimum Euclidean distance-based precoder for MIMO wireless systems," EURASIP Journal on Advances in Signal Processing, vol. 2013, no. 1, article 39, pp. 1-12, 2013.

[21] X. Zhu and R. D. Murch, "Performance analysis of maximum likelihood detection in a MIMO antenna system," IEEE Transactions on Communications, vol. 50, no. 2, pp. 187-191, 2002.

[22] B. Vrigneau, J. Letessier, P. Rostaing, L. Collin, and G. Burel, "Theoretical results about MIMO minimal distance precoder and performances comparison," IEICE Transactions on Communications, vol. 91, no. 3, pp. 821-828, 2008.

[23] DaVinci project, FP7 INFSO-ICT-216203, http://www.ict-davinci-codes.eu/.

[24] E. Sengul, E. Akay, and E. Ayanoglu, "Diversity analysis of single and multiple beamforming," IEEE Transactions on Communications, vol. 54, no. 6, pp. 990-993, 2006.

[25] L. G. Ordoez, D. P. Palomar, A. Pages-Zamora, and J. R. Fonollosa, "High-SNR analytical performance of spatial multiplexing MIMO systems with CSI," IEEE Transactions on Signal Processing, vol. 55, no. 11, pp. 5447-5463, 2007. 

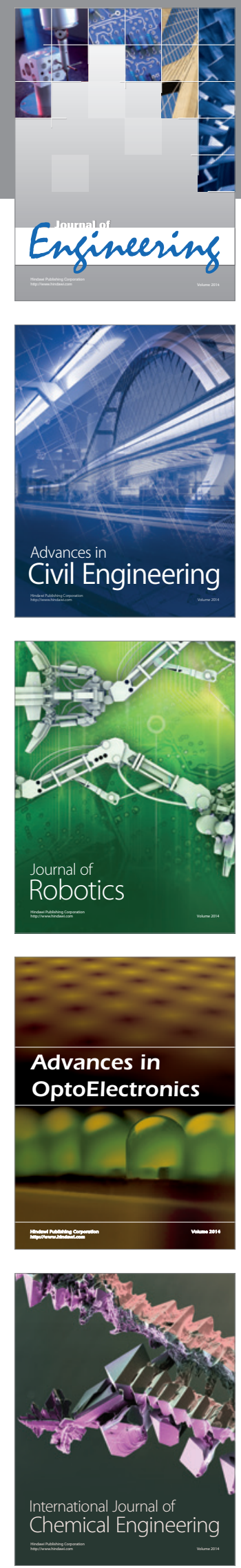

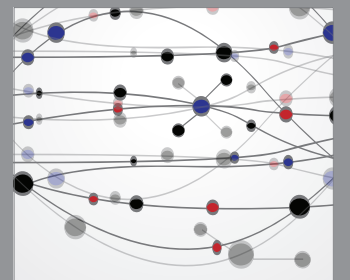

The Scientific World Journal
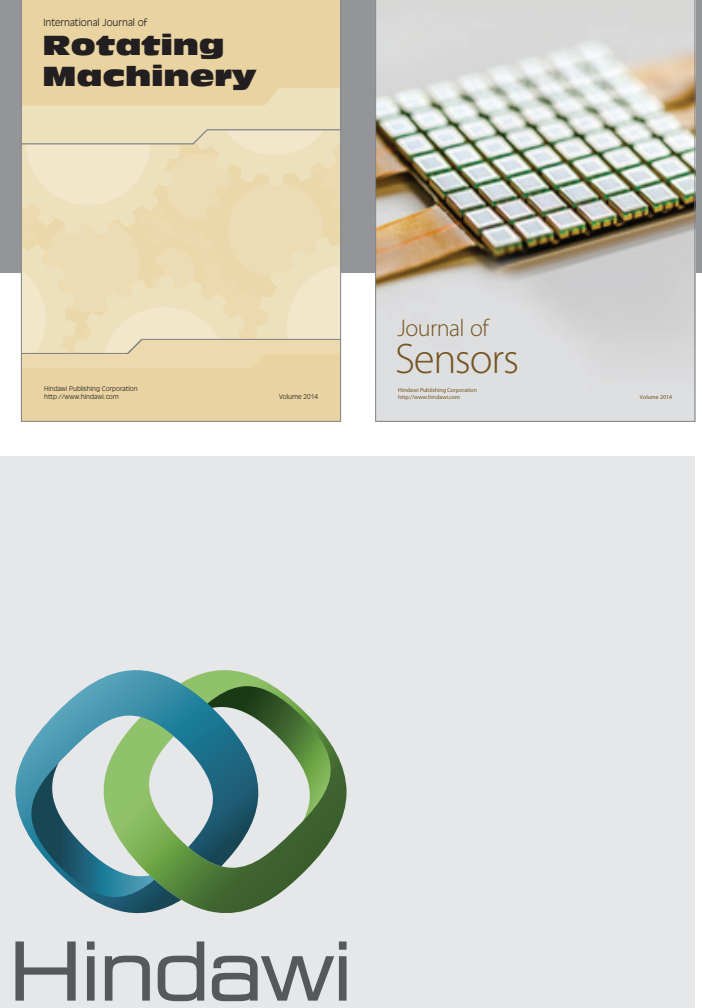

Submit your manuscripts at http://www.hindawi.com
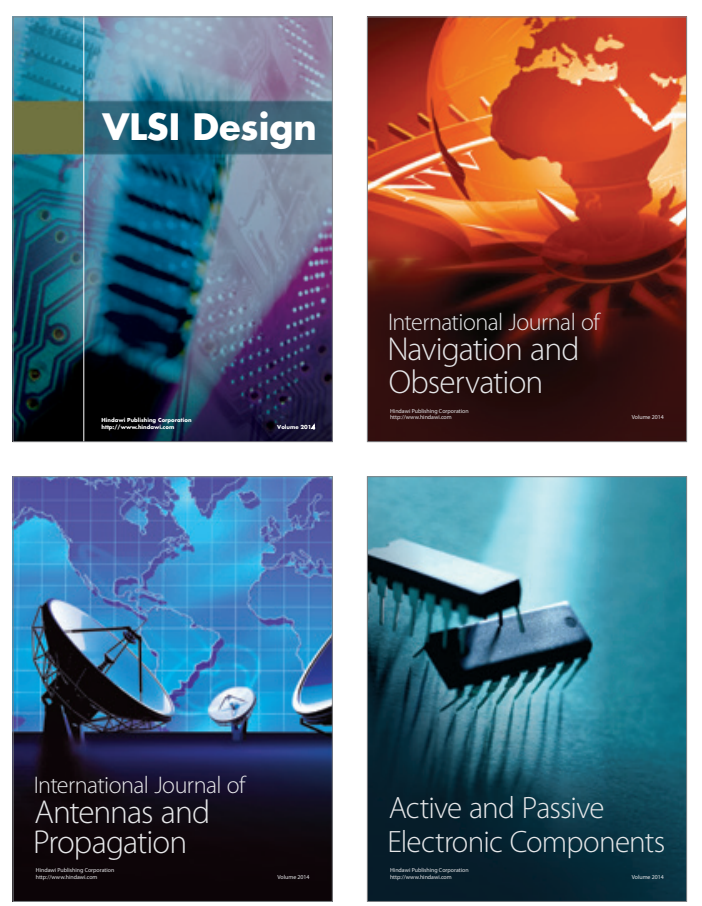
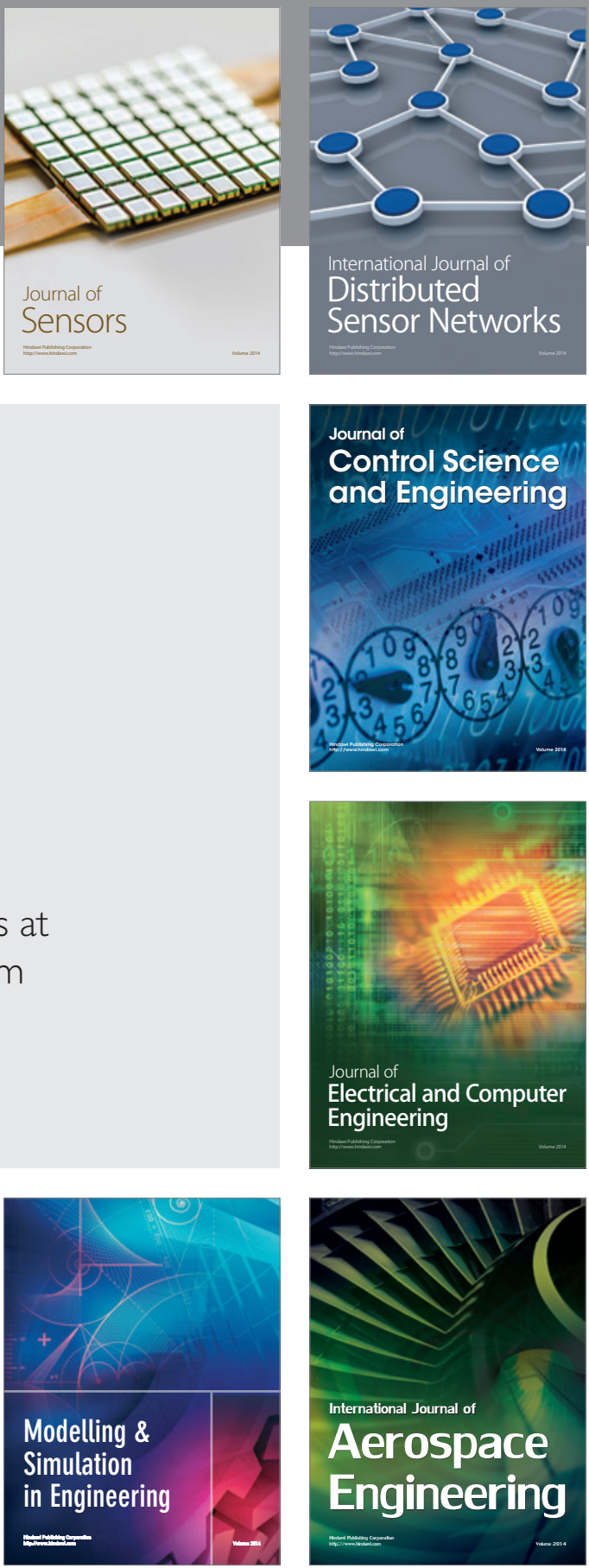

Journal of

Control Science

and Engineering
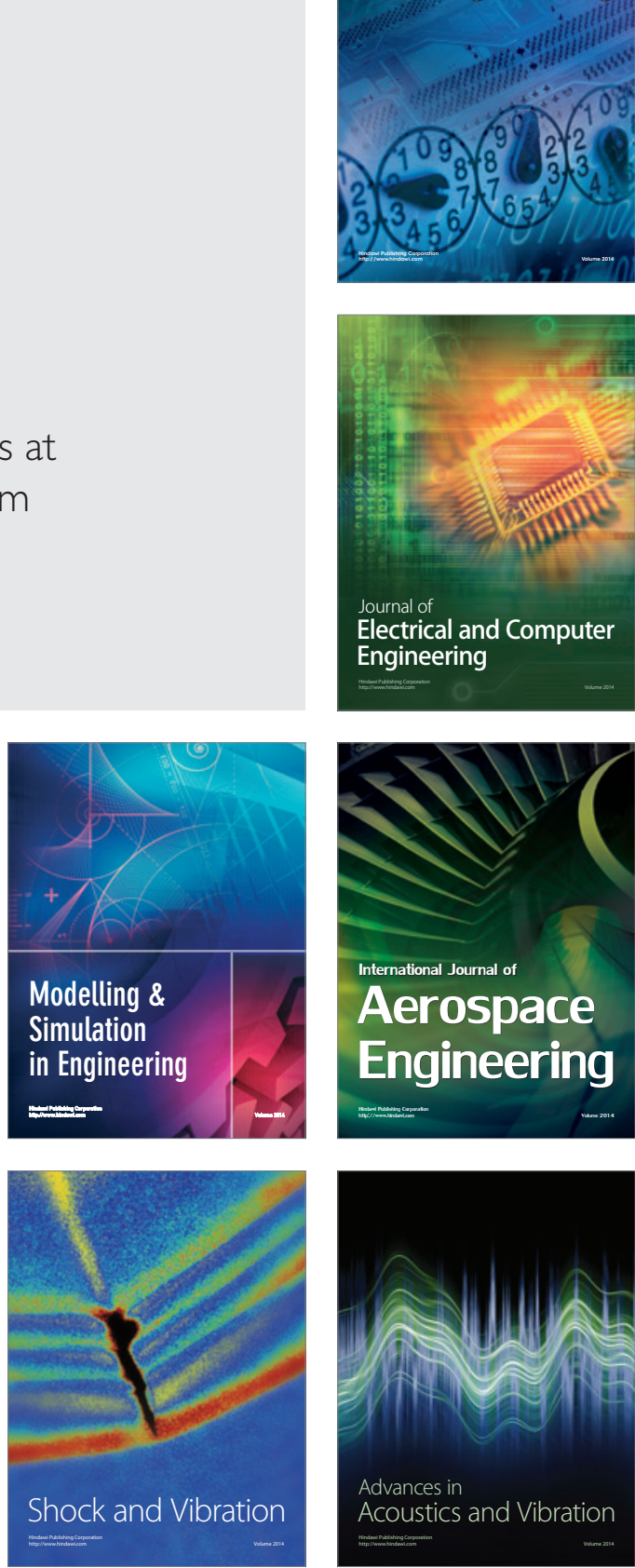